\title{
Cerebrospinal Fluid Biomarkers in Cerebral Amyloid Angiopathy
}

Gargi Banerjee ${ }^{\mathrm{a}}$, Gareth Ambler ${ }^{\mathrm{b}}$, Ashvini Keshavan ${ }^{\mathrm{c}}$, Ross W. Paterson ${ }^{\mathrm{c}}$, Martha S. Foiani ${ }^{\mathrm{d}, \mathrm{e}}$, Jamie Toombs $^{\mathrm{d}, \mathrm{e}}$, Amanda Heslegrave ${ }^{\mathrm{d}, \mathrm{e}}$, John C. Dickson ${ }^{\mathrm{f}}$, Francesco Fraioli ${ }^{\mathrm{f}}$, Ashley M. Groves ${ }^{\mathrm{f}}$, Michael P. Lunn ${ }^{\text {d,g }}$, Nick C. Fox ${ }^{\mathrm{c}, \mathrm{e}}$, Henrik Zetterberg ${ }^{\mathrm{d}, \mathrm{e}, \mathrm{h}, \mathrm{i}}$, Jonathan M. Schott ${ }^{\mathrm{c}, 1}$ and David J. Werring ${ }^{\mathrm{a}, 1, *}$

${ }^{a}$ Stroke Research Centre, Department of Brain Repair and Rehabilitation, UCL Queen Square Institute of Neurology and the National Hospital for Neurology and Neurosurgery, London, UK

${ }^{\mathrm{b}}$ Department of Statistical Science, University College London, Gower Street, London, UK

${ }^{\mathrm{c} D e m e n t i a}$ Research Centre, Department of Neurodegenerative Disease, UCL Queen Square

Institute of Neurology, London, UK

${ }^{\mathrm{d}}$ Department of Neurodegenerative Disease, UCL Queen Square Institute of Neurology, London, UK

${ }^{\mathrm{e}} U K$ Dementia Research Institute at UCL, London, UK

${ }^{\mathrm{f}}$ Institute of Nuclear Medicine, UCL and University College Hospital, London, UK

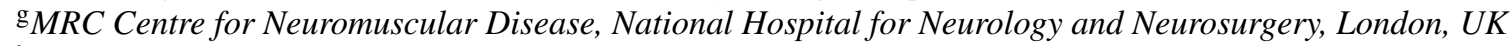

${ }^{\mathrm{h}}$ Clinical Neurochemistry Laboratory, Sahlgrenska University Hospital, Mölndal, Sweden

${ }^{\mathrm{i}}$ Department of Psychiatry and Neurochemistry, Institute of Neuroscience and Physiology,

The Salhgrenska Academy at the University of Gothenburg, Gothenburg, Sweden

Accepted 5 February 2020

\begin{abstract}
.
Background: There is limited data on cerebrospinal fluid (CSF) biomarkers in sporadic amyloid- $\beta$ (A $\beta$ ) cerebral amyloid angiopathy (CAA).

Objective: To determine the profile of biomarkers relevant to neurodegenerative disease in the CSF of patients with CAA. Methods: We performed a detailed comparison of CSF markers, comparing patients with CAA, Alzheimer's disease (AD), and control (CS) participants, recruited from the Biomarkers and Outcomes in CAA (BOCAA) study, and a Specialist Cognitive Disorders Service.

Results: We included 10 CAA, 20 AD, and 10 CS participants (mean age 68.6, 62.5, and 62.2 years, respectively). In unadjusted analyses, CAA patients had a distinctive CSF biomarker profile, with significantly lower $(p<0.01)$ median concentrations of $A \beta_{38}, A \beta_{40}, A \beta_{42}, s A \beta P P \alpha$, and sA $\beta P P \beta$. CAA patients had higher levels of neurofilament light (NFL) than the CS group $(p<0.01)$, but there were no significant differences in CSF total tau, phospho-tau, soluble TREM2 (sTREM2), or neurogranin concentrations. AD patients had higher total tau, phospho-tau and neurogranin than CS and CAA groups. In age-adjusted analyses, differences for the CAA group remained for $A \beta_{38}, A \beta_{40}, A \beta_{42}$, and $\mathrm{sA} \beta P P \beta$. Comparing CAA patients with amyloid-PET positive $(n=5)$ and negative $(n=5)$ scans, PET positive individuals had lower $(p<0.05)$ concentrations of $\mathrm{CSF} A \beta_{42}$, and higher total tau, phospho-tau, NFL, and neurogranin concentrations, consistent with an "AD-like" profile.
\end{abstract}

\footnotetext{
${ }^{1}$ These authors contributed equally to the manuscript.

*Correspondence to: David J. Werring, Stroke Research Centre, Department of Brain Repair and Rehabilitation, UCL Queen
}

Square Institute of Neurology, Russell Square House, 10-12 Russell Square, London WC1B 5EH, UK. Tel.: +44 (0)20 3108 7493; Fax: +44 (0)20 7833 8613; E-mail: d.werring@ucl.ac.uk. 
Conclusion: CAA has a characteristic biomarker profile, suggestive of a global, rather than selective, accumulation of amyloid species; we also provide evidence of different phenotypes according to amyloid-PET positivity. Further replication and validation of these preliminary findings in larger cohorts is needed.

Keywords: Alzheimer's disease, amyloid- $\beta$, biomarkers, cerebral amyloid angiopathy, cerebrospinal fluid

\section{INTRODUCTION}

Sporadic amyloid- $\beta$ (A $\beta)$ cerebral amyloid angiopathy (CAA) can be reliably diagnosed during life using the clinico-radiological Boston criteria $[1,2]$, but nearly all of its associated imaging features are likely to be irreversible markers of late stage disease [3]. Body fluid biomarkers, using either cerebrospinal fluid (CSF) or blood, are of interest as important candidate biomarkers which can be sampled repeatedly, allow for measurement of a variety of different disease-related processes, and provide insights into disease dynamics.

To date, most data on CSF biomarkers in CAA have focused on $A \beta\left(A \beta_{40}, A \beta_{42}\right)$ and tau (total tau, t-tau, and phospho-tau, p-tau) measures. However, there is limited information on other amyloid species in CAA, including smaller proteins such as $A \beta_{38}$, soluble amyloid- $\beta$ protein precursor (sA $\beta P P) \alpha$ and $\beta$. Additionally, a number of newer fluid biomarkers are of potential interest in CAA. Neurofilament light (NFL), soluble TREM2 (sTREM2) and neurogranin are promising new biomarkers for Alzheimer's disease (AD), but it is not clear whether they are specific for parenchymal amyloid.

Our aim was to perform a detailed comparison of amyloid markers $\left(A \beta_{38}, A \beta_{40}, A \beta_{42}, s A \beta P P \alpha\right.$, and $\mathrm{sA} \beta P P \beta)$ and other markers studied in neurodegenerative disease (t-tau, p-tau, NFL, sTREM2, and neurogranin) in the CSF of patients with $\mathrm{AD}$, CAA, and control (CS) participants in an exploratory hypothesis-generating study. To explore the heterogeneity of CAA CSF profile and relationship with parenchymal amyloid deposition, we then performed post-hoc analyses comparing the CSF profiles of CAA patients with amyloid-PET positive and negative scans.

\section{MATERIALS AND METHODS}

\section{Patient selection}

Participants were included from two sources. Firstly, we included participants from the crosssectional prospective observational BOCAA
(Biomarkers and Outcomes in Cerebral Amyloid Angiopathy) study (10 patients with CAA, 5 CS participants). Ethical approval for the BOCAA study was granted in October 2015 by the NHS Health Research Authority London (REC reference 15/LO/1443). Secondly, we included samples collected by the Specialist Cognitive Disorders Service at the National Hospital of Neurology and Neurosurgery (NHNN), University College London Hospitals (UCLH) NHS Trust, London, UK (20 samples from patients with AD, 5 samples from age-matched CS participants). This study was approved by the Regional Ethics Committee at UCL.

In all cases, informed written consent was obtained for each participant, and inclusion criteria were standardized to be consistent with the BOCAA study (further details below).

\section{Patients with CAA}

All patients with CAA were recruited from the BOCAA study [4]. Consecutive patients with CAA were identified from a prospectively collected research database. Patients with CAA all met at least probable modified Boston Criteria [2], and were not included if they had evidence of co-existing $\mathrm{AD}$ or deep perforator (hypertensive) arteriopathy [4]. Further inclusion criteria were: age $\geq 55$ years, Mini-Mental State Examination (MMSE) score $\geq 23$, modified Rankin scale (mRS) $\leq 3$ and capacity to give informed consent. Those with contraindications to PET or MRI scanning or lumbar puncture were excluded.

\section{Control (CS) participants}

CS participants were included from two sources. In all cases, CS participants were required to have no prior history of significant neurological disease. Further inclusion criteria were: age $\geq 55$ years, MMSE score $\geq 23$, and modified Rankin scale $(\mathrm{mRS}) \leq 3$.

Firstly, we included CS participants recruited as part of the BOCAA study, where patient partners were invited to participate as healthy volunteers. CS participants were also identified from a prospectively collected database of patients attending the ambulatory transient ischemic attack (TIA) service, provided 
by the NHNN; patients whose final diagnosis was not stroke, TIA, or any other significant neurological condition (and met the inclusion and exclusion criteria) were invited to participate. Those with contraindications to PET or MRI scanning or lumbar puncture were excluded.

Secondly, we included samples collected by the Specialist Cognitive Disorders Service at the NHNN. Samples for age-matched CS participants were included if their final diagnosis, made on the basis of clinical assessment, imaging, and CSF, was not one of dementia or any other neurodegenerative condition [5]. MR imaging (acquired as part of routine clinical care) was reviewed for evidence of previous infarction (including lacunes), cerebral microbleeds, and cortical superficial siderosis; participant samples were only included in the absence of all of these features. Atrophy (medial temporal [6] and global cortical [7]) and white matter hyperintensities [8] were assessed on brain imaging, and those with evidence of moderate or severe grades of these imaging features were excluded.

\section{Patients with Alzheimer's disease}

Patients with AD presented with "typical" [9] amnestic symptoms, were aged $\geq 55$ years, and had a final diagnosis (on the basis of clinical assessment, imaging, and CSF) that was in keeping with $\mathrm{AD}$; additionally, all imaging was reviewed for the presence of cerebral microbleeds and cortical superficial siderosis, and samples from patients with these features were not included (in order to avoid patients with mixed CAA and AD pathology). The CSF criteria for $\mathrm{AD}$ diagnosis were the presence of a t-tau/A $\beta_{42}$ ratio $>0.88$ together with $A \beta_{42}<630 \mathrm{pg} / \mathrm{ml}$ [5].

\section{CSF analysis}

All CSF analyses were performed by the Biomarker Laboratory of the UK Dementia Research Institute at UCL (Group Lead: Professor Henrik Zetterberg). CSF was collected, processed, and stored according to standardized procedures, and was identical for all diagnostic groups [10]. Samples were collected in polypropylene tubes, immediately transported to the laboratory by hand, and centrifuged (at 1,750 for $5 \mathrm{~min}$ at $4^{\circ} \mathrm{C}$ ) within $30 \mathrm{~min}$ from collection; samples were then aliquoted and stored at $-80^{\circ} \mathrm{C}$ until testing. All biochemical assays were performed by operators blinded to the clinical diagnosis.

\section{Amyloid markers}

$A \beta_{38}, A \beta_{40}$, and $A \beta_{42}$ were measured by electrochemiluminescence (ECL) using a Meso Scale Discovery V-PLEX A $\beta$ peptide panel 1(6E10) kit, according to the manufacturer's instructions (MSD, Rockville, MD). Briefly, samples were diluted 1:2 with diluent 35 and added in duplicate to microplate wells coated with mouse monoclonal peptide specific capture antibodies for human $\mathrm{A} \beta \mathrm{x}-38 / \mathrm{x}-40 / \mathrm{x}-42$. Samples were incubated with anti-A $\beta$ (amino acids 1-16 epitope) antibody (6E10 clone) as the detection antibody conjugated with an electrically excitable SULFO-TAG. Concentrations were calculated from ECL signal using a four-parameter logistic curve fitting method with the MSD Workbench software package. Intra-assay CVs (coefficients of variation) were less than $10 \%$. All samples were measured on the same day by a single operator using the same reagents.

$\mathrm{sA} \beta P P \alpha$ and $\mathrm{sA} \beta P P \beta$ were measured by ECL using a Meso Scale Discovery sA $\beta P P \alpha / s A \beta P P \beta$ Kit, according to the manufacturer's instructions (MSD, Rockville, MD). Briefly, samples were diluted $1: 4$ with $1 \%$ Blocker $\mathrm{A}$ and added in duplicate to microplate wells coated with mouse $(\operatorname{sA} \beta P P \alpha)$ and rabbit (sA $\beta P P \beta)$ monoclonal peptide specific capture antibodies. Samples were incubated with anti-sA $\beta P P \alpha$ and anti-sA $\beta P P \beta$ detection antibodies conjugated with an electrically excitable SULFOTAG. Concentrations were calculated from ECL signal using a four-parameter logistic curve fitting method with the MSD Workbench software package. Intra-assay CVs were less than $20 \%$. All samples were measured on the same day by a single operator using the same reagents.

\section{Tau markers ( $t$-tau and $p$-tau)}

The levels of CSF t-tau and $p$-tau $(181 \mathrm{P})$ were determined using a sandwich ELISA (INNOtest ${ }^{\circledR}$ hTAU-Ag p-Tau $(181 \mathrm{P})$; Fujirebio Europe N.V., Gent, Belgium) constructed to measure both normal tau and phosphorylated tau. Briefly, for the hTAU Ag assay, tau protein is captured from CSF samples by a monoclonal anti-tau antibody (AT120) bound to a microtiter plate. Captured tau is detected with two biotinylated tau-specific monoclonal antibodies (HT7 and BT2). Similarly, for the t-tau assay, $p$-tau $(181 \mathrm{P})$ is captured from CSF samples by anti-tau antibody HT7 bound onto a microtiter plate. Captured $\mathrm{p}-\operatorname{tau}_{(181 \mathrm{P})}$ is detected with a biotinylated monoclonal anti-p-tau antibody (AT270). In both assays, peroxidase-labelled strep- 
tavidin and tetramethylbenzidine (TMB) substrate are also added. Peroxidase-catalyzed hydrolysis produces a colorimetric signal. Sample concentrations are extrapolated from a standard curve, fitted using a 4-parameter logistic algorithm. Intra-assay CVs were less than $20 \%$.

\section{Neurofilament light}

NFL was measured using the commercially available NF-Light ELISA, according to the manufacturer's instructions (UmanDiagnostics, Ume $a$, Sweden). Briefly, samples were diluted 1:2 with sample diluent and added in duplicate to microplate wells coated with a monoclonal capture antibody specific for NFL. Samples were incubated with a biotinylated NFL-specific monoclonal detection antibody. The detection complex was completed with the addition of horseradish peroxidase-labelled streptavidin and TMB substrate. Peroxidase-catalyzed hydrolysis produces a colorimetric signal. Sample concentrations were extrapolated from a standard curve, fitted using a 4-parameter logistic algorithm. Intra-assay CVs were less than $10 \%$. Samples were run on two different days by different operators; the inter-assay $\mathrm{CV}$ was below $16 \%$.

\section{sTREM2}

Samples were analyzed using an immunoassay protocol adapted from a previously published protocol [11]. Streptavidin-coated 96-well plates (Meso-Scale Discovery (MSD), Rockville, MD, USA) were blocked overnight at $4^{\circ} \mathrm{C}$ in block buffer $(0.5 \%$ bovine serum albumin (BSA) and $0.05 \%$ Tween 20 in PBS; pH 7.4). The plates were then incubated with the biotinylated polyclonal goat anti-human TREM2 capture antibody $(0.25 \mu \mathrm{g} / \mathrm{ml}$; BAF1828, R\&D Systems, Minneapolis, MN, USA) diluted in block buffer, shaking for $1 \mathrm{~h}$ at room temperature. They were subsequently washed five times with wash buffer $(0.05 \%$ Tween 20 in PBS) and incubated for $2 \mathrm{~h}$ shaking at room temperature with $50 \mu \mathrm{L}$ per well of either the standard curve constructed from recombinant human TREM2 protein (11084-H08H-50, Sino Biological Inc., Beijing, China) diluted in assay buffer $(0.25 \%$ BSA and $0.05 \%$ Tween 20 in PBS; pH 7.4) to produce concentrations ranging between $4000 \mathrm{pg} / \mathrm{ml}$ and $62.5 \mathrm{pg} / \mathrm{ml}$, or CSF samples diluted 1 in 4 in assay buffer. Standards and CSF samples were assayed in duplicate. Plates were again washed five times with wash buffer before incubation for $1 \mathrm{~h}$ shaking at room temperature with the detection antibody, monoclonal mouse antihuman TREM2 antibody ( $1 \mu \mathrm{g} / \mathrm{ml}$; (B-3): sc373828, Santa Cruz Biotechnology, TX, USA), diluted in block buffer. After five additional washing steps, plates were incubated with the secondary antibody (SULFO-TAG-labelled goat anti-mouse secondary antibody, R32AC-5, MSD) and incubated shaking for $1 \mathrm{~h}$ in the dark. Lastly, plates were washed three times with wash buffer then twice in PBS alone. The electrochemical signal was developed by adding MSD Read buffer T 4× (R92TC-2, MSD) diluted 1 in 2, and the light emission measured using the MSD Sector Imager 6000. The concentration of sTREM2 was calculated using a five-parameter logistic curve fitting method with the MSD Workbench software package. Intra-assay CVs were less than $10 \%$, and all samples were measured on the same day by a single operator using the same reagents.

\section{Neurogranin}

Neurogranin was measured with the EUROIMMUN Elisa (EQ6551-9601-L) according to manufacturer's instructions (EUROIMMUN, Lübeck, Germany). Briefly, samples were incubated with biotinylated monoclonal anti-neurogranin antibody, followed by addition to microplate wells coated with monoclonal antibodies specific for human neurogranin truncated at P75. Finally, streptavidin peroxidase conjugate was added to initiate the colorchanging reaction. Intra-assay $\mathrm{CVs}$ were less than $10 \%$, and all samples were measured on the same day by a single operator using the same reagents. Sample concentrations are extrapolated from a standard curve, fitted using a 5-parameter logistic algorithm.

\section{Amyloid-PET acquisition and interpretation}

All participants in the BOCAA study underwent PET (using the amyloid ligand ${ }^{18}$ F-Florbetapir, Amyvid) and MR scanning, acquired using the same hybrid Siemens Biograph PET/MR scanner; the protocol for acquisition and initial processing has been previously described [12]. Visual reads were performed by a trained individual who was blind to all participant clinical details (including diagnosis).

\section{Statistics}

Statistical analysis was performed using Stata (Version 15.1). Group characteristics were compared using one-way ANOVA (age), chi squared (sex), or 
Kruskal-Wallis (MMSE) tests. Median and interquartile range values were calculated for each biomarker, and, given the non-normal distribution of the data, comparisons between groups were made using the Kruskal-Wallis test. If a significant difference was identified (defined as $\mathrm{p}<0.05$ ), Dunn's test was used for post-hoc comparisons, and a Bonferroni correction (resultant $\mathrm{p}$ value multiplied by 3 ) was applied.

In order to perform age-adjusted analyses, we used quantile regression (comparing group medians) and calculated predicted medians. We then performed post-hoc pairwise comparisons of the ageadjusted medians; statistical significance was defined as Bonferroni-corrected $p<0.05$.

Finally, we performed exploratory post-hoc analyses comparing biomarkers between PET positive and negative CAA patients using Wilcoxon-MannWhitney tests.

\section{RESULTS}

We included 20 patients with $\mathrm{AD}, 10$ patients with CAA, and 10 CS participants in this analysis; baseline characteristics are shown in Table 1. Patients with CAA were older than the two other groups (mean age \pm SD: CAA $68.6 \pm 3.0$ years, AD $62.5 \pm 4.1$ years, and CS $62.2 \pm 5.4$ years). As expected, those in the AD group had a lower MMSE (median score 24, compared with 29 for CAA and CS groups).

Of the patients with CAA, $5(50 \%)$ presented with intracerebral hemorrhage, and the remainder presented with transient focal neurological episodes associated with convexity subarachnoid hemorrhage. All CAA patients had been clinically asymptomatic in the 6 months prior to their study visits. Amongst the CAA group, 7 patients (70\%) showed evidence of cortical superficial siderosis (focal in three cases, disseminated in four cases) and 9 (90\%) had lobar microbleeds (median number, 3.5). All CAA patients showed evidence of both deep and periventricular white matter hyperintensities on MRI (median Fazekas scores, 1 in deep and 2 in periventricular regions [8]).

\section{Individual markers}

\section{Univariable comparisons}

Univariable comparisons are shown in Table 1 and Fig. 1. There were significant differences between the three groups for the following markers: $A \beta_{38}$, $\mathrm{A} \beta_{40}, \mathrm{~A} \beta_{42}, \mathrm{sA} \beta \mathrm{PP} \alpha, \mathrm{sA} \beta \mathrm{PP} \beta, \mathrm{t}$-tau, p-tau, NFL, and neurogranin. There was no significant difference in sTREM2 levels between the three groups.

In post-hoc comparisons (Table 1, Fig. 1), patients with CAA had significantly lower CSF levels of $A \beta_{38}, A \beta_{40}, A \beta_{42}, s A \beta P P \alpha$ and sA $\beta P P \beta$ than both the AD and CS groups. Patients with AD had lower CSF $A \beta_{42}$ than the CS group, but this was not statistically significant after Bonferroni correction; there were no significant differences between the AD and $\mathrm{CS}$ groups for the other amyloid markers. For the tau markers (t-tau, p-tau), there were no statistically significant differences between the CAA and CS groups; patients with $\mathrm{AD}$ had significantly higher levels than both CAA and CS group. Patients with both CAA and AD had significantly higher CSF NFL than the CS group; there was no statistically significant difference between the CAA and $\mathrm{AD}$ groups. There was no difference in neurogranin levels between CAA and either the $\mathrm{AD}$ or the $\mathrm{CS}$ groups; patients with $\mathrm{AD}$ had significantly higher levels of CSF neurogranin than the CS group.

\section{Age-adjusted quantile regression}

Results from the age-adjusted quantile regression are shown in Table 2; scatter plots demonstrating the distribution of each biomarker by age are shown in Supplementary Figure 1. For the amyloid markers, there were significant differences between the three groups for $A \beta_{38}, A \beta_{40}, A \beta_{42}$, and $\mathrm{A} A \mathrm{PP} \beta$, but not sA $\beta P P \alpha$. Pairwise comparisons of the age-adjusted medians found significant differences between CAA and $A D$ groups for $A \beta_{38}$ (higher in $A D$ group; median difference $1,480 \mathrm{pg} / \mathrm{ml}$ ), $A \beta_{40}$ (higher in $\mathrm{AD}$ group; median difference $3,540 \mathrm{pg} / \mathrm{ml}$ ) and $\mathrm{sA} \beta P P \beta$ (higher in AD group; median difference $48.6 \mathrm{pg} / \mathrm{ml}$ ); for $A \beta_{42}$, the difference between the CAA and $A D$ groups did not reach statistical significance (higher in $\mathrm{AD}$ group; median difference $162 \mathrm{pg} / \mathrm{ml}$ ). There were significant differences between the CAA and CS groups for $A \beta_{38}$ (higher in CS group; median difference $1,650 \mathrm{pg} / \mathrm{ml}$ ), $A \beta_{40}$ (median difference $4060 \mathrm{pg} / \mathrm{ml}$ ), $\mathrm{A} \beta_{42}$ (higher in CS group; median difference $394 \mathrm{pg} / \mathrm{ml}$ ), and $\mathrm{sA} \beta \mathrm{PP} \beta$ (higher in CS group; median difference $58.8 \mathrm{pg} / \mathrm{ml}$ ). For $\mathrm{A} \beta_{42}$, there was a significant difference between the $\mathrm{AD}$ and $\mathrm{CS}$ groups (higher in CS group; median difference $232 \mathrm{pg} / \mathrm{ml}$ ).

There were significant differences between the three groups for both t-tau and p-tau. Pairwise comparisons of the age-adjusted medians found significant differences for both t-tau (higher in $\mathrm{AD}$ group; median difference $357 \mathrm{pg} / \mathrm{ml}$ ) and $\mathrm{p}$-tau (higher in AD group; median difference $40.9 \mathrm{pg} / \mathrm{ml}$ ) 


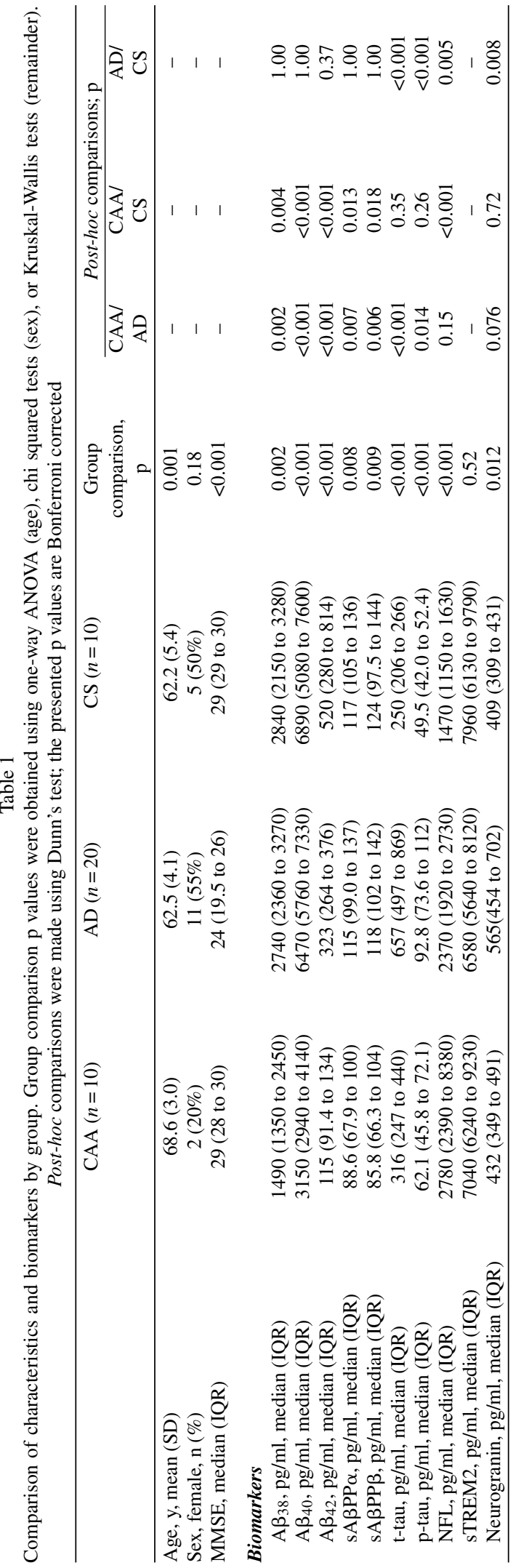



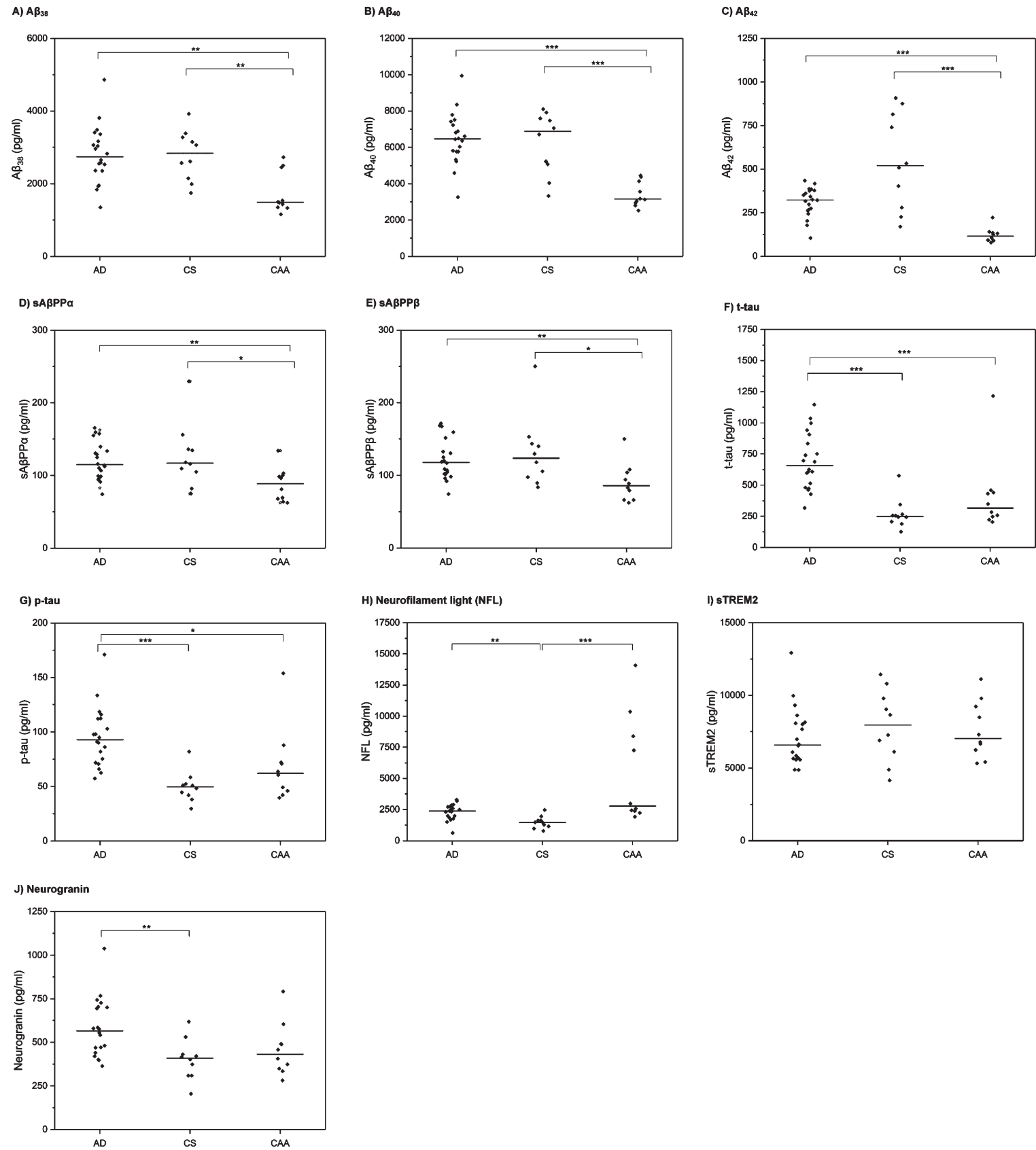

Fig. 1. CSF biomarker profiles in AD, CAA, and control (CS) participants. Horizontal line indicates median value per group. Each diamond indicates an individual data point. $p$-values are derived from ost-hoc Dunn's test and have been Bonferroni-corrected. * $p \leq 0.05$, ** $p \leq 0.01$, $* * * p \leq 0.001$.

between the CAA and AD groups. There was no significant difference between the CAA and CS groups for either $\mathrm{t}$-tau (median difference $53.1 \mathrm{pg} / \mathrm{ml}$ ) or p-tau (median difference $2.2 \mathrm{pg} / \mathrm{ml}$ ). There were significant differences between the AD and CS groups for t-tau (higher in AD group; median difference
$410 \mathrm{pg} / \mathrm{ml}$ ) and $\mathrm{p}$-tau (higher in $\mathrm{AD}$ group; median difference $43.1 \mathrm{pg} / \mathrm{ml}$ ).

There was a significant difference in neurogranin between the three groups, but no significant differences were identified in pairwise post-hoc comparisons of the adjusted medians. There were no 
Table 2

Age-adjusted quantile regression (comparing medians)

\begin{tabular}{|c|c|c|c|}
\hline Biomarker & Group & $\begin{array}{c}\text { Age-adjusted } \\
\text { difference } \\
\text { in medians (SE) }\end{array}$ & $\mathrm{p}$ \\
\hline $\mathrm{A} \beta_{38}, \mathrm{pg} / \mathrm{ml}$ & $\begin{array}{l}\text { CAA } \\
\mathrm{AD} \\
\mathrm{CS}\end{array}$ & $\begin{array}{c}\text { Reference group } \\
1480(370) \\
1650(417)\end{array}$ & $<0.001$ \\
\hline $\mathrm{A} \beta_{40}, \mathrm{pg} / \mathrm{ml}$ & $\begin{array}{l}\text { CAA } \\
\mathrm{AD} \\
\mathrm{CS}\end{array}$ & $\begin{array}{c}\text { Reference group } \\
3540(846) \\
4060(954)\end{array}$ & $<0.001$ \\
\hline $\mathrm{A} \beta_{42}, \mathrm{pg} / \mathrm{ml}$ & $\begin{array}{l}\text { CAA } \\
\mathrm{AD} \\
\mathrm{CS}\end{array}$ & $\begin{array}{c}\text { Reference group } \\
162(83.9) \\
394(94.5)\end{array}$ & $<0.001$ \\
\hline $\mathrm{sA} \beta \mathrm{PP} \alpha, \mathrm{pg} / \mathrm{ml}$ & $\begin{array}{l}\text { CAA } \\
\mathrm{AD} \\
\mathrm{CS}\end{array}$ & $\begin{array}{c}\text { Reference group } \\
18.9(16.1) \\
20.0(18.1)\end{array}$ & 0.47 \\
\hline $\mathrm{sA} \beta \mathrm{PP} \beta, \mathrm{pg} / \mathrm{ml}$ & $\begin{array}{l}\text { CAA } \\
\mathrm{AD} \\
\mathrm{CS}\end{array}$ & $\begin{array}{c}\text { Reference group } \\
48.6(19.1) \\
58.8(21.5)\end{array}$ & 0.024 \\
\hline $\mathrm{t}$-tau, $\mathrm{pg} / \mathrm{ml}$ & $\begin{array}{l}\text { CAA } \\
\mathrm{AD} \\
\mathrm{CS}\end{array}$ & $\begin{array}{c}\text { Reference group } \\
357(115) \\
-53.1(129)\end{array}$ & $<0.001$ \\
\hline $\mathrm{p}$-tau, $\mathrm{pg} / \mathrm{ml}$ & $\begin{array}{l}\text { CAA } \\
\mathrm{AD} \\
\mathrm{CS}\end{array}$ & $\begin{array}{c}\text { Reference group } \\
40.9(12.6) \\
-2.20(14.2)\end{array}$ & $<0.001$ \\
\hline $\mathrm{NFL}, \mathrm{pg} / \mathrm{ml}$ & $\begin{array}{l}\text { CAA } \\
\mathrm{AD} \\
\mathrm{CS}\end{array}$ & $\begin{array}{c}\text { Reference group } \\
-511(844) \\
-1310(952)\end{array}$ & 0.36 \\
\hline sTREM2, pg/ml & $\begin{array}{l}\text { CAA } \\
\mathrm{AD} \\
\mathrm{CS}\end{array}$ & $\begin{array}{c}\text { Reference group } \\
-76.9(1270) \\
1100(1430)\end{array}$ & 0.55 \\
\hline Neurogranin, pg/ml & $\begin{array}{l}\text { CAA } \\
\text { AD } \\
\text { CS }\end{array}$ & $\begin{array}{c}\text { Reference group } \\
210(91.5) \\
24.0(103)\end{array}$ & 0.021 \\
\hline
\end{tabular}

significant differences between the three groups for NFL or STREM2.

\section{A $\beta$ ratios}

Results for comparison of $A \beta$ ratios are provided in the Supplementary Material.
Associations with amyloid-PET positivity

We then performed post-hoc analyses comparing the CSF profiles of CAA patients with amyloidPET positive and negative scans. Half of the patients in the CAA group $(n=5 ; 50 \%)$ were PET positive by visual read. There were no differences in age, sex or MMSE score between the PET positive and negative groups. When comparing CSF biomarkers (Table 3; Supplementary Figure 3), PET positive CAA patients had lower CSF levels of $A \beta_{42}$ than PET negative CAA patients (median 92.5 versus $134 \mathrm{pg} / \mathrm{ml}, p=0.047$ ), and higher CSF t-tau (median 440 versus $247 \mathrm{pg} / \mathrm{ml}, p=0.016)$, $\mathrm{p}$-tau (median 72.1 versus $45.8 \mathrm{ng} / \mathrm{ml}, p=0.009$ ), NFL (median 8380 versus $2390 \mathrm{pg} / \mathrm{ml}, p=0.016$ ), and neurogranin (median 491 versus $349 \mathrm{pg} / \mathrm{ml}, p=0.016$ ).

Given these results, we performed a further comparison, in which amyloid-PET negative CAA patients were compared with $\mathrm{AD}$ and CS groups; these results are provided in the Supplementary Material. Patients with amyloid-PET negative CAA had lower $A \beta_{38}, A \beta_{40}$, and $A \beta_{42}$ than both $A D$ and $C S$ groups, with no statistically significant differences between the $\mathrm{AD}$ and $\mathrm{CS}$ groups for these markers. There were no significant differences in $\mathrm{sA} \beta \mathrm{PP} \alpha$ and sA $\beta P P \beta$ between the three groups; the results for $\mathrm{t}-$ tau, p-tau, NFL, and sTREM2 were similar to those identified in the original analyses. In contrast to the original analysis, neurogranin in amyloid-PET negative CAA was significantly lower than the AD group.

\section{DISCUSSION}

In this exploratory, hypothesis-generating study, we found that patients with CAA had a distinc-

Table 3

Comparison of PET positive and negative patients with CAA. $p$-values were obtained using one-way ANOVA (age), chi squared (sex) tests, or Wilcoxon-Mann-Whitney tests (remainder)

\begin{tabular}{|c|c|c|c|}
\hline & PET positive $(n=5)$ & PET negative $(n=5)$ & $p$ \\
\hline Age, y, mean (SD) & $69.4(3.1)$ & $67.8(2.9)$ & 0.43 \\
\hline Sex, female, $n(\%)$ & $1(20.0)$ & $1(20.0)$ & 1.00 \\
\hline MMSE, median (IQR) & $29(29$ to 29$)$ & $29(28$ to 30$)$ & 0.83 \\
\hline $\mathrm{A} \beta_{38}, \mathrm{pg} / \mathrm{ml}$, median (IQR) & $2450(1350$ to 2500$)$ & $1480(1440$ to 1500$)$ & 0.46 \\
\hline $\mathrm{A} \beta_{40}, \mathrm{pg} / \mathrm{ml}$, median (IQR) & $4140(2800$ to 4370$)$ & $3130(3040$ to 3170$)$ & 0.60 \\
\hline $\mathrm{A} \beta_{42}, \mathrm{pg} / \mathrm{ml}$, median (IQR) & $92.5(89.4$ to 105$)$ & $134(131$ to 140$)$ & 0.047 \\
\hline $\mathrm{sA} \beta \mathrm{PP} \alpha, \mathrm{pg} / \mathrm{ml}$, median (IQR) & $81.0(67.9$ to 96.2$)$ & $98.5(69.4$ to 100$)$ & 0.60 \\
\hline $\mathrm{sA} \beta \mathrm{PP} \beta, \mathrm{pg} / \mathrm{ml}$, median (IQR) & $82.8(79.0$ to 104$)$ & $88.7(66.3$ to 94.1$)$ & 0.75 \\
\hline t-tau, pg/ml, median (IQR) & 440 (431 to 458$)$ & 247 (222 to 258$)$ & 0.016 \\
\hline p-tau, pg/ml, median (IQR) & $72.1(70.8$ to 87.8$)$ & $45.8(42.1$ to 49.1$)$ & 0.009 \\
\hline NFL, pg/ml, median (IQR) & $8380(7260$ to 10400$)$ & $2390(2230$ to 2440$)$ & 0.016 \\
\hline sTREM2, pg/ml, median (IQR) & $8500(7300$ to 9780$)$ & $6660(5420$ to 6780$)$ & 0.12 \\
\hline Neurogranin, pg/ml, median (IQR) & 491 (489 to 604$)$ & 349 (334 to 373$)$ & 0.016 \\
\hline
\end{tabular}


tive CSF profile compared with CS participants and patients with $\mathrm{AD}$. In unadjusted analyses, patients with CAA showed lower levels of all amyloid components measured $\left(A \beta_{38}, A \beta_{40}, A \beta_{42}, s A \beta P P \alpha\right.$, and sA $\beta P P \beta$ ) and higher NFL, but did not show differences in CSF t-tau, p-tau, sTREM2, or neurogranin profile. Patients with $\mathrm{AD}$ had higher t-tau, p-tau and neurogranin than both control participants and patients with CAA. In age-adjusted analyses, differences for the CAA group remained for $A \beta_{38}$, $A \beta_{40}, A \beta_{42}$, and sA $\beta P P \beta$. Finally, we performed exploratory post-hoc comparisons within the CAA group, comparing those with amyloid-PET positive and negative scans, and found that those who were PET positive showed differences in $\mathrm{A} \beta_{42}$, t-tau, $p$-tau, NFL, and neurogranin, in an AD-like profile.

Our results for $A \beta_{40}, A \beta_{42}$, t-tau, and p-tau in CAA are in keeping with previously reported data [13-19]; however, we extend this earlier work further by demonstrating that $A \beta_{40}$ and $A \beta_{42}$ are not the only amyloid species to be reduced in CAA. The processing pathway from $A \beta P P$ to pathological $A \beta$ is well described [20-22]. However, CAA differs from $\mathrm{AD}$ in that parenchymal $\mathrm{A} \beta$ plaques are predominantly composed of $A \beta_{42}$, whereas the vascular $A \beta$ deposits in CAA are a mixture of $A \beta_{40}$ and $A \beta_{42}$, with the former being more common $[16,23]$. The reduced levels of CSF $A \beta_{40}$ and $A \beta_{42}$ previously described in CAA are thought to be secondary to "selective trapping" of both these species in the vasculature, in contrast with $\mathrm{AD}$, where only $A \beta_{42}$ is found ("trapped") in the parenchyma [16]. A $\beta$ peptides of other lengths, including $A \beta_{38}$, have also been shown to be deposited in the leptomeningeal vasculature [24]. Our finding of reductions in CSF $A \beta_{38}$ and sA $\beta P P \beta$ (and $s A \beta P P \alpha$, in our unadjusted analyses) are novel, and support the protein elimination failure hypothesis for CAA [25], which proposes that CAA results due to failed $A \beta$ clearance via intramural periarterial drainage pathways [26]. Our results might suggest that a range of $A \beta P P$ and $A \beta$ elements are trapped within the cerebral vasculature, potentially the result of a generalized (rather than selective) protein clearance failure. An alternative interpretation is that the reduction in all $A \beta P P$ and $A \beta$ species we measured reflects decreased $A \beta P P$ expression, processing and release, rather than altered clearance of the proteins.

Our results also provide new information on nonamyloid biomarkers in CAA. We found significant elevations in t-tau, $\mathrm{p}$-tau, and neurogranin in patients with $\mathrm{AD}$, but not in patients with CAA compared with healthy controls. This is in contrast with other studies which have found that $\mathrm{t}$-tau and $\mathrm{p}$-tau levels in CAA are higher than controls but lower than patients with $\mathrm{AD}[13,16,18,27]$, and may reflect our small sample size. Pathological aggregation of tau protein is important in $\mathrm{AD}[28,29]$; tau aggregation is thought to result in synaptic dysfunction and subsequent neuronal loss, and in $\mathrm{AD}$, it is tau (rather than $A \beta$ ) pathology that most closely correlates with cognition [29]. The intermediate tau levels described in CAA might thus be reflective of coexistent $\mathrm{AD}$ pathology. Cognitive impairment is a recognized feature of CAA [30] and while there is evidence that CAA is associated with atrophy (presumably secondary to neuronal loss [31]), cognitive impairment in these patients might be secondary to other mechanisms, such as network disruption [32] or impaired blood flow responses [33]. Our CSF findings suggest that synaptic dysfunction is a less prominent feature of CAA compared with $\mathrm{AD}$, and that these markers might be useful for distinguishing these two $A \beta$ pathologies in patients with cognitive impairment.

We also provide new data on other markers that have not been tested in CAA. NFL has shown great promise as a biomarker in a large number of neurological conditions [34], including sporadic cerebral small vessel disease [35-37], although age-adjusted analyses were only performed in one study [36]. We did not find a difference between the AD, CAA, and CS groups in age-adjusted analyses for CSF NFL, but in unadjusted analyses, the CAA group had higher NFL than the control participant group, and in the post-hoc analyses, patients with CAA who were amyloid-PET positive had particularly high levels, raising the possibility that this is a useful marker for co-existing $\mathrm{AD}$ and CAA pathology. As seems to be the case for many other conditions, including cerebral small vessel disease [37], NFL in CAA is likely to find utility as a marker of the intensity of neurodegeneration and so a useful measure of prognosis rather than diagnosis, particularly as it can be measured in the serum; further work is needed to investigate this. We did not find any differences in sTREM2 between the $\mathrm{AD}, \mathrm{CAA}$, or control groups; this is in contrast with previous studies, in which STREM2 was found to be elevated in the CSF of AD patients [38-42]. Again, our inability to identify a statistically significant difference might result from our relatively small sample size.

Our exploratory post-hoc analyses comparing amyloid-PET positive and negative patients with CAA raise new questions about the interactions 
between $\mathrm{AD}$ and CAA pathologies. Although the group sizes are small (with only five participants per group), these findings confirm that not all patients with neuroimaging evidence of CAA are PET positive (in keeping with previous reports; a recent metaanalysis found that amyloid-PET sensitivity in CAA ranged from $60 \%$ to $91 \%$ ) [43], and suggest that there is a sub-group with an AD-like profile-lower CSF $A \beta_{42}$, higher CSF t-tau, phospho-tau, NFL, and neurogranin - who have evidence of additional fibrillary amyloid deposition. As described above, the marked increase in NFL in PET positive CAA patients might suggest that NFL is particularly sensitive for dual $\mathrm{AD}$ and CAA pathology. Neurogranin was able to discriminate between PET negative CAA, $\mathrm{AD}$, and CS groups, with elevations only evident in the $\mathrm{AD}$ group, providing further evidence that this might be a very specific marker for fibrillary $A D$ pathology. Together, these data support the hypothesis that amyloid-PET positivity in CAA is a measure of co-existent parenchymal $A \beta$ rather than a measure of vascular amyloid and raises the possibility that the degree or extent of co-existent AD pathology in CAA can be measured using these CSF markers. An alternative explanation is that these markers, together with amyloid-PET positivity, are indicative of a higher vascular amyloid burden that results in neurodegeneration via non-AD mechanisms. Further work in larger cohorts is needed, both to replicate these findings and explore these hypotheses further.

Our work has a number of strengths. We were able to evaluate a large number of markers and to reduce false-positive error we have used two separate statistical methods, one rank-based (Kruskal-Wallis) and the other an age-adjusted analysis based upon comparison of medians. However, there are some limitations. As mentioned earlier, this was a small exploratory study which may not have been powered to detect differences for all the biomarkers considered. Given our small sample size, we acknowledge the possibility of false-positive results due to multiple comparisons as a limitation of our work. While larger studies have considered some of the CSF markers we describe (in particular $A \beta_{40}, A \beta_{42}$, t-tau, and $p$-tau), we provide new data in CAA for a number of other markers, which we hope will inform future research with regard to effect sizes and sample size calculations. We acknowledge that there can be discrepancies between clinical and pathological diagnoses of CAA and AD, as well as significant overlap in these pathologies. While we attempted to avoid this on the basis of clinical and radiological findings, we did not have autopsy data, which would be the gold standard. However, we would argue that most clinicians who review patients with $\mathrm{AD}$ or CAA make their diagnosis on the basis of clinical and radiological (rather than autopsy) findings, and therefore our results remain of relevance. We specifically selected non-demented CAA patients with "early" or mild disease; these patients may not have such marked biomarker perturbations as those with more severe disease. The AD patients selected from the Specialist Cognitive Disorders Service may not be fully representative of $\mathrm{AD}$ patients more generally; this is a highly specialist tertiary service, which often sees younger patients or those with atypical presentations. We used CSF criteria (t-tau and $A \beta_{42}$ ) in order to identify patients with $\mathrm{AD}$ and half of the control group, and so these markers are not fully independent in our analyses. Many of our control participants presented to neurology services (either the TIA clinic, or the Specialist Cognitive Disorders Service), and although we only included individuals without clinical, radiological, or CSF evidence of significant neurological disease, we acknowledge that they cannot be described as true "healthy" controls; this is a further limitation of our work. We selected control participants with minimal radiological evidence of brain pathology (WMH, atrophy), and this may not be truly representative of unselected age-matched individuals without $\mathrm{AD}$ or CAA. We determined PET positivity on qualitative (i.e., based on visual reads) rather than quantitative grounds, and the group sizes are particularly small in this post-hoc analysis, which precludes from ageadjustment. However, despite these limitations, we provide important new data on these body fluid markers in CAA, and in particular provide data on effect sizes that will be critical for determining sample sizes for larger future studies.

\section{Conclusions}

Our findings suggest that patients with CAA appear to have a CSF profile distinct from both patients with $\mathrm{AD}$ and age-matched control participants, characterized by a global reduction in secreted $\mathrm{A} \beta \mathrm{PP}$ and $\mathrm{A} \beta$ species, but normal neuronal protein levels (tau, neurogranin) compared with AD patients. Replication of these findings in larger cohorts with longitudinal cohort measurements is required to confirm these markers as effective biomarkers for CAA, and their value for monitoring disease progression in clinical trials. 


\section{ACKNOWLEDGMENTS}

We would like to acknowledge our consultant colleagues from the Dementia Research Centre, who contributed patient samples, and the clinicians who performed the lumbar punctures.

The BOCAA study was funded by the Rosetrees Trust. GB receives funding from the NIHR (as an Academic Clinical Fellow) and the Rosetrees Trust. AK acknowledges the support of the Wolfson Foundation and Weston Brain Institute. HZ is a Wallenberg Academy Fellow supported by grants from the Swedish Research Council, the European Research Council and the UK Dementia Research Institute at UCL. JMS acknowledges the support of the National Institute for Health Research University College London Hospitals Biomedical Research Centre, Wolfson Foundation, EPSRC (EP/J020990/1), MRC Dementias Platform UK (MR/L023784/1), ARUK (ARUK-Network 2012-6ICE; ARUK-PG2017-1946; ARUK-PG2017-1946), Brain Research UK (UCC14191), Weston Brain Institute (UB170045) and European Union's Horizon 2020 research and innovation programme (Grant 666992); in addition NCF acknowledges support from the UK Dementia Research Institute. DJW receives research support from the Stroke Association, the British Heart Foundation and the Rosetrees Trust. This work was undertaken at UCLH/UCL which receives a proportion of funding from the Department of Health's National Institute for Health Research (NIHR) Biomedical Research Centres funding scheme.

Authors' disclosures available online (https:// www.j-alz.com/manuscript-disclosures/19-1254r2).

\section{SUPPLEMENTARY MATERIAL}

The supplementary material is available in the electronic version of this article: https://dx.doi.org/ 10.3233/JAD-191254.

\section{REFERENCES}

[1] Knudsen KA, Rosand J, Karluk D, Greenberg SM (2001) Clinical diagnosis of cerebral amyloid angiopathy: Validation of the Boston criteria. Neurology 56, 537-539.

[2] Linn J, Halpin A, Demaerel P, Ruhland J, Giese AD, Dichgans M, van Buchem MA, Bruckmann H, Greenberg SM (2010) Prevalence of superficial siderosis in patients with cerebral amyloid angiopathy. Neurology 74, 1346-1350.
[3] Greenberg SM, Al-Shahi Salman R, Biessels GJ, van Buchem M, Cordonnier C, Lee JM, Montaner J, Schneider JA, Smith EE, Vernooij M, Werring DJ (2014) Outcome markers for clinical trials in cerebral amyloid angiopathy. Lancet Neurol 13, 419-428.

[4] Banerjee G, Werring DJ (2019) Feasibility of clinical trial recruitment for cerebral amyloid angiopathy: A specialist single centre experience. J Neurol Sci 409, 116580.

[5] Weston PS, Paterson RW, Modat M, Burgos N, Cardoso MJ, Magdalinou N, Lehmann M, Dickson JC, Barnes A, Bomanji JB, Kayani I, Cash DM, Ourselin S, Toombs J, Lunn MP, Mummery CJ, Warren JD, Rossor MN, Fox NC, Zetterberg H, Schott JM (2015) Using florbetapir positron emission tomography to explore cerebrospinal fluid cut points and gray zones in small sample sizes. Alzheimers Dement (Amst) 1, 440-446.

[6] Scheltens P, Leys D, Barkhof F, Huglo D, Weinstein HC, Vermersch P, Kuiper M, Steinling M, Wolters EC, Valk J (1992) Atrophy of medial temporal lobes on MRI in "probable" Alzheimer's disease and normal ageing: Diagnostic value and neuropsychological correlates. J Neurol Neurosurg Psychiatry 55, 967-972.

[7] Pasquier F, Leys D, Weerts JG, Mounier-Vehier F, Barkhof F, Scheltens P (1996) Inter- and intraobserver reproducibility of cerebral atrophy assessment on MRI scans with hemispheric infarcts. Eur Neurol 36, 268-272.

[8] Fazekas F, Kleinert R, Offenbacher H, Schmidt R, Kleinert G, Payer F, Radner H, Lechner H (1993) Pathologic correlates of incidental MRI white matter signal hyperintensities. Neurology 43, 1683-1689.

[9] Elahi FM, Miller BL (2017) A clinicopathological approach to the diagnosis of dementia. Nat Rev Neurol 13, 457-476.

[10] Blennow K, Hampel H, Weiner M, Zetterberg H (2010) Cerebrospinal fluid and plasma biomarkers in Alzheimer disease. Nat Rev Neurol 6, 131-144.

[11] Kleinberger G, Yamanishi Y, Suarez-Calvet M, Czirr E, Lohmann E, Cuyvers E, Struyfs H, Pettkus N, WenningerWeinzierl A, Mazaheri F, Tahirovic S, Lleo A, Alcolea D, Fortea J, Willem M, Lammich S, Molinuevo JL, SanchezValle R, Antonell A, Ramirez A, Heneka MT, Sleegers K, van der Zee J, Martin JJ, Engelborghs S, Demirtas-Tatlidede A, Zetterberg H, Van Broeckhoven C, Gurvit H, Wyss-Coray T, Hardy J, Colonna M, Haass C (2014) TREM2 mutations implicated in neurodegeneration impair cell surface transport and phagocytosis. Sci Transl Med 6, 243 ra286.

[12] Lane CA, Parker TD, Cash DM, Macpherson K, Donnachie E, Murray-Smith H, Barnes A, Barker S, Beasley DG, Bras J, Brown D, Burgos N, Byford M, Jorge Cardoso M, Carvalho A, Collins J, De Vita E, Dickson JC, Epie N, Espak M, Henley SMD, Hoskote C, Hutel M, Klimova J, Malone IB, Markiewicz P, Melbourne A, Modat M, Schrag A, Shah S, Sharma N, Sudre CH, Thomas DL, Wong A, Zhang H, Hardy J, Zetterberg H, Ourselin S, Crutch SJ, Kuh D, Richards M, Fox NC, Schott JM (2017) Study protocol: Insight 46 - a neuroscience sub-study of the MRC National Survey of Health and Development. BMC Neurol 17, 75.

[13] Renard D, Castelnovo G, Wacongne A, Le Floch A, Thouvenot E, Mas J, Gabelle A, Labauge P, Lehmann S (2012) Interest of CSF biomarker analysis in possible cerebral amyloid angiopathy cases defined by the modified Boston criteria. J Neurol 259, 2429-2433.

[14] Tamura R, Tomita H, Mizutani K, Miwa T (2014) The importance of amyloid beta protein in cerebrospinal fluid when you recognize convexal subarachnoid hemorrhage. Eur Neurol 71, 283-287. 
[15] Garcia Estevez DA, Garcia-Dorrego RM, Nieto-Baltar B, Marey-Garrido M, Hierro-Torner T (2017) Spontaneous convexity subarachnoid haemorrhage: Clinical series of 3 patients with associated cerebral amyloid angiopathy. $\mathrm{Neu}$ rologia 32, 213-218.

[16] Verbeek MM, Kremer BP, Rikkert MO, Van Domburg PH, Skehan ME, Greenberg SM (2009) Cerebrospinal fluid amyloid beta(40) is decreased in cerebral amyloid angiopathy. Ann Neurol 66, 245-249.

[17] Martinez-Lizana E, Carmona-Iragui M, Alcolea D, GomezChoco M, Vilaplana E, Sanchez-Saudinos MB, Clarimon J, Hernandez-Guillamon M, Munuera J, Gelpi E, GomezAnson B, de Juan-Delago M, Delgado-Mederos R, Montaner J, Ois A, Amaro S, Blesa R, Marti-Fabregas J, Lleo A, Fortea J (2015) Cerebral amyloid angiopathyrelated atraumatic convexal subarachnoid hemorrhage: An ARIA before the tsunami. J Cereb Blood Flow Metab 35, 710-717.

[18] Charidimou A, Friedrich JO, Greenberg SM, Viswanathan A (2018) Core cerebrospinal fluid biomarker profile in cerebral amyloid angiopathy: A meta-analysis. Neurology $\mathbf{9 0 ,}$ e754-e762.

[19] van Etten ES, Verbeek MM, van der Grond J, Zielman R, van Rooden S, van Zwet EW, van Opstal AM, Haan J, Greenberg SM, van Buchem MA, Wermer MJ, Terwindt GM (2017) beta-Amyloid in CSF: Biomarker for preclinical cerebral amyloid angiopathy. Neurology 88, 169-176.

[20] Kummer MP, Heneka MT (2014) Truncated and modified amyloid-beta species. Alzheimers Res Ther 6, 28.

[21] Selkoe DJ (1991) The molecular pathology of Alzheimer's disease. Neuron 6, 487-498.

[22] Hardy JA, Higgins GA (1992) Alzheimer's disease: The amyloid cascade hypothesis. Science 256, 184-185.

[23] Iwatsubo T, Odaka A, Suzuki N, Mizusawa H, Nukina N, Ihara Y (1994) Visualization of A beta 42(43) and A beta 40 in senile plaques with end-specific A beta monoclonals: Evidence that an initially deposited species is A beta 42(43). Neuron 13, 45-53.

[24] Kakuda N, Miyasaka T, Iwasaki N, Nirasawa T, WadaKakuda S, Takahashi-Fujigasaki J, Murayama S, Ihara Y, Ikegawa M (2017) Distinct deposition of amyloidbeta species in brains with Alzheimer's disease pathology visualized with MALDI imaging mass spectrometry. Acta Neuropathol Commun 5, 73.

[25] Carare RO, Hawkes CA, Jeffrey M, Kalaria RN, Weller RO (2013) Review: Cerebral amyloid angiopathy, prion angiopathy, CADASIL and the spectrum of protein elimination failure angiopathies (PEFA) in neurodegenerative disease with a focus on therapy. Neuropathol Appl Neurobiol 39, 593-611.

[26] Albargothy NJ, Johnston DA, MacGregor-Sharp M, Weller RO, Verma A, Hawkes CA, Carare RO (2018) Convective influx/glymphatic system: Tracers injected into the CSF enter and leave the brain along separate periarterial basement membrane pathways. Acta Neuropathol 136, 139-152.

[27] Renard D, Gabelle A, Hirtz C, Demattei C, Thouvenot E, Lehmann S (2016) Cerebrospinal fluid Alzheimer's disease biomarkers in isolated supratentorial cortical superficial siderosis. J Alzheimers Dis 54, 1291-1295.

[28] Ballatore C, Lee VM, Trojanowski JQ (2007) Tau-mediated neurodegeneration in Alzheimer's disease and related disorders. Nat Rev Neurosci 8, 663-672.

[29] Ittner A, Ittner LM (2018) Dendritic tau in Alzheimer's disease. Neuron 99, 13-27.
[30] Banerjee G, Carare R, Cordonnier C, Greenberg SM, Schneider JA, Smith EE, Buchem MV, Grond JV, Verbeek MM, Werring DJ (2017) The increasing impact of cerebral amyloid angiopathy: Essential new insights for clinical practice. J Neurol Neurosurg Psychiatry 88, 982-994.

[31] Fotiadis P, van Rooden S, van der Grond J, Schultz A, Martinez-Ramirez S, Auriel E, Reijmer Y, van Opstal AM, Ayres A, Schwab KM, Alzheimer's Disease Neuroimaging I, Hedden T, Rosand J, Viswanathan A, Wermer M, Terwindt G, Sperling RA, Polimeni JR, Johnson KA, van Buchem MA, Greenberg SM, Gurol ME (2016) Cortical atrophy in patients with cerebral amyloid angiopathy: A case-control study. Lancet Neurol 15, 811-819.

[32] Reijmer YD, Fotiadis P, Martinez-Ramirez S, Salat DH, Schultz A, Shoamanesh A, Ayres AM, Vashkevich A, Rosas D, Schwab K, Leemans A, Biessels GJ, Rosand J, Johnson KA, Viswanathan A, Gurol ME, Greenberg SM (2015) Structural network alterations and neurological dysfunction in cerebral amyloid angiopathy. Brain 138, 179-188.

[33] Peca S, McCreary CR, Donaldson E, Kumarpillai G, Shobha N, Sanchez K, Charlton A, Steinback CD, Beaudin AE, Fluck D, Pillay N, Fick GH, Poulin MJ, Frayne R, Goodyear BG, Smith EE (2013) Neurovascular decoupling is associated with severity of cerebral amyloid angiopathy. Neurology 81, 1659-1665.

[34] Zetterberg H (2016) Neurofilament light: A dynamic crossdisease fluid biomarker for neurodegeneration. Neuron $\mathbf{9 1}$, 1-3.

[35] Jonsson M, Zetterberg H, van Straaten E, Lind K, Syversen S, Edman A, Blennow K, Rosengren L, Pantoni L, Inzitari D, Wallin A (2010) Cerebrospinal fluid biomarkers of white matter lesions - cross-sectional results from the LADIS study. Eur J Neurol 17, 377-382.

[36] Duering M, Konieczny MJ, Tiedt S, Baykara E, Tuladhar AM, Leijsen EV, Lyrer P, Engelter ST, Gesierich B, Achmuller M, Barro C, Adam R, Ewers M, Dichgans M, Kuhle J, de Leeuw FE, Peters N (2018) Serum neurofilament light chain levels are related to small vessel disease burden. J Stroke 20, 228-238.

[37] Gattringer T, Pinter D, Enzinger C, Seifert-Held T, Kneihsl M, Fandler S, Pichler A, Barro C, Grobke S, Voortman M, Pirpamer L, Hofer E, Ropele S, Schmidt R, Kuhle J, Fazekas F, Khalil M (2017) Serum neurofilament light is sensitive to active cerebral small vessel disease. Neurology 89, 21082114.

[38] Gispert JD, Suarez-Calvet M, Monte GC, Tucholka A, Falcon C, Rojas S, Rami L, Sanchez-Valle R, Llado A, Kleinberger G, Haass C, Molinuevo JL (2016) Cerebrospinal fluid sTREM2 levels are associated with gray matter volume increases and reduced diffusivity in early Alzheimer's disease. Alzheimers Dement 12, 1259-1272.

[39] Heslegrave A, Heywood W, Paterson R, Magdalinou N, Svensson J, Johansson P, Ohrfelt A, Blennow K, Hardy J, Schott J, Mills K, Zetterberg H (2016) Increased cerebrospinal fluid soluble TREM2 concentration in Alzheimer's disease. Mol Neurodegener 11, 3.

[40] Piccio L, Deming Y, Del-Aguila JL, Ghezzi L, Holtzman DM, Fagan AM, Fenoglio C, Galimberti D, Borroni B, Cruchaga C (2016) Cerebrospinal fluid soluble TREM2 is higher in Alzheimer disease and associated with mutation status. Acta Neuropathol 131, 925-933.

[41] Suarez-Calvet M, Kleinberger G, Araque Caballero MA, Brendel M, Rominger A, Alcolea D, Fortea J, Lleo A, Blesa R, Gispert JD, Sanchez-Valle R, Antonell A, Rami L, 
Molinuevo JL, Brosseron F, Traschutz A, Heneka MT, Struyfs H, Engelborghs S, Sleegers K, Van Broeckhoven C, Zetterberg H, Nellgard B, Blennow K, Crispin A, Ewers M, Haass C (2016) sTREM2 cerebrospinal fluid levels are a potential biomarker for microglia activity in early-stage Alzheimer's disease and associate with neuronal injury markers. EMBO Mol Med 8, 466-476.

[42] Suarez-Calvet M, Araque Caballero MA, Kleinberger G, Bateman RJ, Fagan AM, Morris JC, Levin J, Danek A,
Ewers M, Haass C (2016) Early changes in CSF sTREM2 in dominantly inherited Alzheimer's disease occur after amyloid deposition and neuronal injury. Sci Transl Med $\mathbf{8}$, 369 ra178.

[43] Charidimou A, Farid K, Baron JC (2017) Amyloid-PET in sporadic cerebral amyloid angiopathy: A diagnostic accuracy meta-analysis. Neurology $\mathbf{8 9}, 1490-1498$. 